\title{
Iron stable isotope variation of marine organisms in Northwest Pacific Ocean
}

\author{
NANAKO HASEGAWA ${ }^{1}$, TAKAAKI ITAI ${ }^{1}$, TATSUYA \\ KUNISUE $^{2}$, SHINSUKE TANABE ${ }^{2}$ AND YOSHIO \\ TAKAHASHI ${ }^{1}$
}

${ }^{1}$ The University of Tokyo

${ }^{2}$ Ehime University

Presenting Author: n-hasegawa@eps.s.u-tokyo.ac.jp

Despite its essential role, availability of $\mathrm{Fe}$ in marine environment is often poor due to the low solubility of inorganic species. Since migratory predators relies on the trophic transfer as the available Fe source from primary production, establishing the tracer of this process is important. Here we show the variation of $\delta^{56} \mathrm{Fe}$ of marine organisms including fishes (4 species, $\mathrm{n}=19$ ), cephalopod ( 1 species, $\mathrm{n}=1)$, crustacean zooplankton ( 1 pooled sample collected by plankton net), and cetaceans ( 3 species, $n=11$ ) collected around Northwest Pacific Ocean.

All the wild samples were archived in environmental specimen bank, Ehime University, Japan. The muscular $\delta^{56} \mathrm{Fe}$ was analyzed by MC-ICP-MS (Neptune, Thermo Fisher Scientific) after modified column purification protocol by Maréchal et al. (1999). The accuracy of the $\delta^{56} \mathrm{Fe}$ measurements was evaluated through the measurements of biological CRMs (DORM-4, and ERM-CE464). The differences between measured and certified values were $<0.06 \%$ while the precision of duplicate measurements was $0.09 \%$. We obtained new $\delta^{56} \mathrm{Fe}$ value for BCR-414 and DOLT-5 as $-0.09 \pm 0.07 \%$ and $-2.32 \pm 0.07 \%$, respectively.

The variation of muscular $\delta^{56} \mathrm{Fe}$ of marine organisms were as following; zooplankton: $-0.03 \pm 0.08 \%$, fish muscle: $-2.64 \%$ to $-0.71 \%$ ( $\mathrm{n}=19$, average: $-1.65 \%$ ), and cetacean muscle: $-2.37 \%$ o to $-1.74 \%$ o $(n=9$, average: $-2.15 \%$ ). The variation was predominantly controlled by species rather than size and region. The $\delta^{56} \mathrm{Fe}$ generally increased in order of cetaceans < fishes $<$ zooplankton, suggesting that light isotopes tend to enrich with increasing trophic level. However, Japanese sardines (Sardinops melanostictus), an important prey for migratory fish, showed significantly lower values $(-2.64 \%$ to $-1.73 \%$ ) than the higher predator fishes indicating that sequential depletion of heavy isotope is not only the predominant fractionation process. Species specific difference of intestinal iron absorption rate or the ratio of non-heme $\mathrm{Fe} /$ heme $\mathrm{Fe}$ in whole body are the possible factors controlling muscular $\delta^{56} \mathrm{Fe}$ variation. Hence, $\delta^{56} \mathrm{Fe}$ can be a tool to trace trophic transfer of $\mathrm{Fe}$ through marine ecosystem. 